\title{
Heamocoagulase Agkistrodon on Hemostatic Effectivity on Posterior Lumbar Operation
}

\author{
Feng Hao*, Chen Huanshi, Bai Ruifei, Li Wei \\ Department of Orthopedics, Liuzhou Municipal Liutie Central Hospital, Liuzhou, China \\ Email address: \\ fenghao79@163.com (Feng Hao) \\ ${ }^{*}$ Corresponding author
}

\section{To cite this article:}

Feng Hao, Chen Huanshi, Bai Ruifei, Li Wei. Heamocoagulase Agkistrodon on Hemostatic Effectivity on Posterior Lumbar Operation. Clinical Medicine Research. Vol. 9, No. 6, 2020, pp. 136-139. doi: 10.11648/j.cmr.20200906.13

Received: December 16, 2020; Accepted: December 22, 2020; Published: December 28, 2020

\begin{abstract}
Objective: Perioperative bleeding volume of posterior spinal surgery is relatively large. This study was aimed to evaluate the hemostatic effect of Hemocoagulase agkistrodon for injection on open lumbar spine surgery. Methods: In this study, a prospective, blinded, randomized, and controlled clinical trial was used to observe the hemostatic effect of $2 \mathrm{U}$ intravenous injection of Hemocoagulase agkistrodon 15 minutes before surgery on the posterior lumbar open surgery. A total of 60 cases were included in this experimental study, 30 cases in the study group (2U agkistrodon hemagglutinin for intravenous injection 15 minutes before surgery) and 30 cases in the control group (Inject normal saline 15 minutes before surgery). We use SAS software to simulate and generate random codes. The third-party blinding method is adopted, that is, a specialized nurse is assigned to allocate study drugs in the order of random codes and intravenously according to the plan. We statistically analyzed the bleeding volume and postoperative drainage volume of posterior lumbar spine surgery, and the changes of coagulation indexes in the two groups. Results: All the selected patients successfully completed the operation, their wounds healed and were discharged from the hospital, and no complications such as infection and thrombosis occurred during the hospitalization. The two groups of patients' coagulation function indicators include prothrombin time (PT), thrombin time (TT), activated partial thromboplastin time (APTT), fibrinogen quantification (FIB), and D2 polymer. The difference was not statistically significant. However, comparing the intraoperative blood loss, $24 \mathrm{~h}$ postoperative drainage, and postoperative total drainage between the two groups, the experimental group was better than the control group. Conclusion: Intravenous injection of Agkistrodon hemagglutinin $2 \mathrm{U}$ 15 minutes before surgery can have a satisfactory hemostatic effect on the posterior lumbar spine surgical incision. It can not only significantly reduce the amount of bleeding from surgical incisions, but also has no significant effect on the body's coagulation function. It is a safe and effective hemostatic drug.
\end{abstract}

Keywords: Heamocoagulase Agkistrodon, Hemostasis, Open Spinal Surgery, Lumbar Degeneration

\section{Introduction}

Heamocoagulase Agkistrodon for injection (trade name: $\mathrm{Su}$ Ling) is a thrombin isolated and purified from the venom of the common snake species Agkistrodon in South China [1]. It is officially approved by SFDA on September 22, 2008, and is now on the domestic market on March 25, 2009 in the national Class I new drug (National Medicine Standard H20080633). Hemocoagulase agkistrodon for injection is developed by using the venom in the snake species unique to my country-Agkistrodon acutus (commonly known as "Five step snake") [2]. Preclinical studies have confirmed that the enzyme can shorten the blood clotting time [2] and bleeding time after tail trimming in mice [3]. It has a good hemostatic effect, and does not affect the number of prothrombin and platelets in the blood [4]. There is no platelet aggregation in normal blood vessels, and there is no risk of thrombosis. Domestic multi-center clinical studies have confirmed that the drug is effective and safety in hemostasis for abdominal surgery [5, 6], thyroid surgery [7], obstetrics and gynecology surgery [4], breast surgery [8], and gynecological surgeries [3]. However, studies have shown that it has no obvious hemostatic effect on chest surgical incisions [9]. This subject aims to evaluate the effectiveness and safety of the drug on the hemostatic effect of posterior lumbar spine surgical incisions. 


\section{Methods}

\subsection{Clinical Data}

Sixty patients who were diagnosed with lumbar spine-related diseases and required surgery in the outpatient department of our hospital from January 2017 to December 2019 were selected. After admission, they were coded according to the outpatient number and randomly divided into study and control groups, with 30 cases in each group. Inclusion criteria: (1) Surgical incision length $8-10 \mathrm{~cm}$; (2) 18-75 years old, no gender limit; (3) Preoperative examination of liver and kidney function is normal; (4) Cagulation function and whole blood viscosity are basically normal; (5) The fasting blood sugar of diabetic patients is controlled at below $8.1 \mathrm{mmol} / \mathrm{L}$, the blood pressure of hypertensive patients is controlled at about $140 / 90 \mathrm{mmHg}$, and cardiac color Doppler ultrasound shows that the left ventricular systolic function is in the normal range. Exclusion criteria: (1) Age $<18$ years or $>75$ years; (2) Have a history of thrombosis and use anticoagulant drugs in the past one month; (3) Have a history of cerebral hemorrhage and other basic hemorrhagic diseases (4) Diabetes patients with fasting blood above $11.0 \mathrm{mmol} / \mathrm{L}$, high In blood pressure patients, systolic blood pressure $>180 \mathrm{mmHg}, \quad$ diastolic blood pressure $>110 \mathrm{mmHg}$; (5) Abnormal coagulation function; (6) Abnormal liver and kidney function; (7) Blood routine showed $\mathrm{WBC}<3 \times 109 / \mathrm{L}$ or $>\mathrm{WBC}<3 \times / 109 / \mathrm{L}$, platelet $<50 \times 109 / \mathrm{L}$; (8) Have a history of severe allergies in the past.

\subsection{Research Method}

30 cases in the study group were injected with Hemocoagulase agkistrodon $2 \mathrm{U}$ intravenously 15 minutes before operation and 30 cases in the control group were injected with normal saline 15 minutes before operation. All patients were in the prone position and intubated under general anesthesia. Posterior lumbar laminectomy, spinal decompression, pedicle screw internal fixation, intervertebral disc removal, cage implantation, and bone graft fusion were performed by the same team of doctors. For obvious venous or arterial bleeding during the operation, use monopolar electrosurgical knife or bipolar electrocoagulation to stop the bleeding. At the end of the operation, a wound drainage tube is routinely placed and not clamped. We observe and record the drainage volume at $24 \mathrm{~h}$ after the operation. The drainage volume is $\leq 30-50 \mathrm{~mL}$, then removed the drainage tube.

\subsection{Observation Indicators}

(1) Intraoperative blood loss (dry gauze weighing method + negative pressure suction barrel reabsorbed blood); (2) $24 \mathrm{~h}$ postoperative wound drainage, postoperative total drainage; (3) $24 \mathrm{~h}$ coagulation before and after Functional indicators [prothrombin time (PT), thrombin time (TT), activated partial thromboplastin time (APTT), fibrinogen quantification (FIB)], D-2 polymer.

\subsection{Statistical Methods}

SPSS 27.0 statistical software was used for data analysis. Measurement data were expressed as " $\mathrm{x} \pm \mathrm{s}$ ". The Chi-square test was used for intra-group comparison, and the independent sample $\mathrm{T}$ test was used for inter-group comparison. $\mathrm{P}<0.05$ was considered statistically significant.

\section{Results}

\subsection{Comparison of Patient Clinical Data}

There was no statistically significant difference in gender, age, height, and body weight between the experimental group and the control group. See Table 1.

Table 1. Comparison of patient clinical data.

\begin{tabular}{llll}
\hline Group & Gender (male /female) & Age (years) & Height (CM) \\
\hline Control $(\mathrm{n}=30)$ & $15 / 15$ & $53.25 \pm 12.85$ & $164.35 \pm 7.36$ \\
Experiment $(\mathrm{n}=30)$ & $16 / 14$ & $55.38 \pm 11.25$ & $60.52 \pm 8.32$ \\
\hline
\end{tabular}

\subsection{Comparison of Clinical Results}

The experimental group and the control group had statistically significant differences in intraoperative blood loss, $24 \mathrm{~h}$ postoperative drainage, and postoperative total drainage.

Table 2. Comparison of intraoperative blood loss and postoperative drainage volume between the two groups $(x \pm s, m L)$.

\begin{tabular}{llll}
\hline Group & Intraoperative blood loss & Postoperative 24h drainage & Postoperative total drainage \\
\hline Control $(\mathrm{n}=30)$ & $420.20 \pm 48.35$ & $135.52 \pm 26.54$ & $310.66 \pm 38.35$ \\
Experiment $(\mathrm{n}=30)$ & $210.20 \pm 85.26$ & $36.52 \pm 22.35$ & $108.52 \pm 29.47$ \\
\hline
\end{tabular}

\subsection{Comparison of Coagulation Indicators}

There was no significant difference in coagulation function indexes PT, APTT, TT, FIB between the two groups before operation, and there was no significant difference between the first day and the third day after operation. There was no statistically significant difference in D-2 polymer between the two groups of patients before operation, and there was no significant difference in D-2 polymer changes between the 1 st day and the 3 rd day after the operation. See Table 3. 
Table 3. Comparison of coagulation indicators between the two groups.

\begin{tabular}{lllllll}
\hline Group & Time & PT & APTT & TT & D2 polymer & FIB \\
\hline \multirow{3}{*}{ Control (n=30) } & Pre-operation & $13.25 \pm 0.76$ & $38.23 \pm 3.52$ & $18.23 \pm 0.52$ & $232.20 \pm 41.52$ & $2.82 \pm 0.48$ \\
& Post-operation (24h) & $14.14 \pm 0.55$ & $39.02 \pm 4.12$ & $19.05 \pm 0.45$ & $225.35 \pm 48.14$ & $3.35 \pm 0.32$ \\
& Post-operation (72h) & $13.83 \pm 0.84$ & $40.08 \pm 3.50$ & $18.74 \pm 0.66$ & $238.35 \pm 38.50$ & $2.99 \pm 0.66$ \\
Experiment & Pre-operation & $13.08 \pm 0.92$ & $39.39 \pm 3.88$ & $18.54 \pm 0.72$ & $250.20 \pm 35.85$ & $3.00 \pm 0.41$ \\
$(\mathrm{n}=30)$ & Post-operation (24h) & $13.75 \pm 0.25$ & $38.58 \pm 3.88$ & $19.07 \pm 0.38$ & $242.02 \pm 44.48$ & $2.69 \pm 0.38$ \\
& Post-operation (72h) & $14.46 \pm 0.22$ & $40.06 \pm 2.92$ & $18.68 \pm 0.55$ & $255.30 \pm 40.28$ & $2.88 \pm 0.48$ \\
\hline
\end{tabular}

\subsection{Clinical Effect}

Both the experimental group and the control group completed the operation successfully. There were no adverse events such as wound infection and thrombosis during the hospitalization. The ultrasound examination of the lower limb blood vessels before discharge from the hospital showed no thrombosis.

\section{Discussions}

Lumbar degeneration is a common cause of low back and leg pain [10]. The vast majority of back and leg pain caused by lumbar degeneration can be cured by conservative treatment [11], and approximately $10 \%$ of patients require surgical treatment. The main surgical methods are minimally invasive and open surgical methods, and the PLIF technique is one of the most classic surgical methods to treat lumbar degeneration [12]. This technique removes the intervertebral disc tissue, fully decompresses, and fuses the intervertebral bones [13]. Most patients obtain satisfactory results. However, exposing the surgical incision through the posterior approach requires stripping of a large number of muscles and exposing cancellous bone, which is likely to cause a lot of bleeding [14]. Therefore, large trauma and bleeding are the significant disadvantages of PLIF surgery. A large amount of blood loss during the perioperative period can lead to postoperative anemia in patients and affect postoperative recovery and wound healing. People with severe anemia even need blood transfusions to improve their anemia [15]. Therefore, reducing perioperative blood loss caused by open lumbar spine surgery can promote the recovery of patients, which is in line with our concept of rapid recovery.

Hemocoagulase agkistrodon for injection is an effective drug to reduce perioperative bleeding [16]. It is a hemostatic active ingredient extracted from the venom of Agkistrodon acutus. At present, the mechanism of action of this hemagglutinase is well known [17]. It mainly splits the A peptide fragment of fibrinogen to form fibrin monomer $(\mathrm{aBBr}) 2$ and polymerizes it in the form of non-covalent cross-linking [7]. Easily soluble fibrin polymer plays a hemostatic effect [18]. In this study, there was no statistically significant difference in the changes of D-2 polymer before and after operation in the experimental group, and there was no significant difference in D-2 polymer before and after operation in the control group. Hemocoagulase agkistrodon does not activate coagulation factor X III, therefore, it is not easy to cause thrombosis. At present, multiple research results indicated that Hemocoagulase agkistrodon was safe and effective for hemostasis by intravenous administration [7, 16]. Relevant animal experiments also confirmed that Hemocoagulase agkistrodon does not affect the number of prothrombin and platelets in the blood, and there is no platelet aggregation in normal blood vessels. Therefore, Hemocoagulase agkistrodon is not easy to cause thrombosis in normal blood vessels [19]. In this study, the method of preoperative intravenous administration was used to observe the effect of Hemocoagulase agkistrodon in spinal surgery. From the results of the study, it can significantly reduce intraoperative bleeding and postoperative wound drainage. At the same time, Hemocoagulase agkistrodon does not affect the blood coagulation function of patients. The results of this study are the same as those of other clinical studies [20].

There are two main problems facing future research. On the one hand, it is the source of natural snake venom. Most of the venomous snake species that can be extracted and used are national protected species [21]. Some snake venoms need to be imported due to the lack of snake species in the country, which is expensive. While incubating the viper technology, we hope to use genetic engineering to produce snake venom thrombin. On the other hand, although Hemocoagulase agkistrodon for injection uses the world's leading snake venom monomer purification technology, the purity of a single component is more than $99 \%$, and it is the only single-component product on the market in my country that has completed all amino acid sequencing. Snake venom thrombin, however, the current research on the structure of snake venom thrombin is mainly focused on the primary structure and secondary structure [22], and there is still a lack of research on higher structure at home and abroad.

\section{Conclusions}

There is a lot of blood loss during and after open lumbar spine surgery, which is likely to cause persistent anemia and affect the patient's recovery. Hemocoagulase agkistrodon for injection has been proved to be a good hemostatic agent in vivo and in vitro after years of clinical and animal experiments. The hemostatic effect is safe and reliable. The results of this study show that preoperative intravenous injection of Hemocoagulase agkistrodon has no significant effect on the body's coagulation function. It can reduce blood loss during surgery and postoperative blood loss. It is a safe and effective hemostatic drug. All in all, the application of Hemocoagulase agkistrodon before open surgery for lumbar degeneration is worthy of clinical application. 


\section{Conflict of Interest}

All the authors do not have any possible conflicts of interest.

\section{Acknowledgements}

This work was supported by Self-financing scientific research project of Guangxi Zhuang Autonomous Region Health and Family Planning Commission (No. Z20170030).

\section{References}

[1] Lin CY, Zhang J, Yang X, Cheng J, Sun LD, Jiang M: Use of thromboelastography to monitor effects of the hemocoagulase on the blood coagulation in patients after thoracic surgery. Ann Palliat Med 2020, 9 (4): 2090-2095.

[2] Li HX, Huang Y, Wu X, Wu T, Cao Y, Wang QM, Qiu YC, Fu WM, Zhang Q, Pang JX: Effects of hemocoagulase agkistrodon on the coagulation factors and its procoagulant activities. Drug Des Dev Ther 2018, 12: 1385-1398.

[3] Wei JM, Zhu MW, Zhang ZT, Jia ZG, He XD, Wan YL, Wang S, Xiu DR, Tang Y, Li J et al: A multicenter, phase III trial of hemocoagulase Agkistrodon: hemostasis, coagulation, and safety in patients undergoing abdominal surgery. Chin Med $J$ (Engl) 2010, 123 (5): 589-593.

[4] Zhang H: The Effects of Hemocoagulase on Coagulation Factors in an Elderly Patient with Upper Gastrointestinal Hemorrhage: A Case Report. Curr Drug Saf 2019, 14 (3): 230-232.

[5] $\mathrm{Xu} \quad \mathrm{YY}, \mathrm{Ma} \mathrm{XH}, \quad$ Zhang SJ: Hemocoagulase agkistrodon-induced anaphylactic shock: A case report and literature review. Int J Clin Pharm Th 2016, 54 (2): 129-134.

[6] Zhu M, Cao J, Jia Z, Duan Z, Liu G, Wei J, Long H: [Hemocoagulase in abdominal operation and its effect on hemoagglutination]. Zhonghua Wai Ke Za Zhi 2002, 40 (8): 581-584.

[7] Yao YT, Yuan X, Fang NX: Hemocoagulase reduces postoperative bleeding and blood transfusion in cardiac surgical patients A PRISMA-compliant systematic review and meta-analysis. Medicine 2019, 98 (52).

[8] Lu X, Yang X, Zhu M, Hua B, Niu X, Xiao W, Wei J: Hemostatic Effect of Hemocoagulase Agkistrodon on Surgical Wound in Breast Cancer Surgery. Zhongguo Yi Xue Ke Xue Yuan Xue Bao 2017, 39 (2): 183-187.

[9] Zeng Z, Xiao P, Chen J, Wei Y: Are batroxobin agents effective for perioperative hemorrhage in thoracic surgery? A systematic review of randomized controlled trials. Blood Coagul Fibrinolysis 2009, 20 (2): 101-107.

[10] Staartjes VE, Quddusi A, Klukowska AM, Schroder ML: Initial classification of low back and leg pain based on objective functional testing: a pilot study of machine learning applied to diagnostics. Eur Spine J 2020, 29 (7): 1702-1708.

[11] Modic MT, Ross JS: Lumbar degenerative disk disease. Radiology 2007, 245 (1): 43-61.

[12] Yang LH, Liu W, Li J, Zhu WY, An LK, Yuan S, Ke H, Zang L: Lumbar decompression and lumbar interbody fusion in the treatment of lumbar spinal stenosis A systematic review and meta-analysis. Medicine 2020, 99 (27).

[13] Yi P, Tang X, Yang F, Tan M: A retrospective controlled study protocol of transforaminal lumbar interbody fusion compared with posterior lumbar interbody fusion for spondylolisthesis. Medicine (Baltimore) 2020, 99 (44): e22878.

[14] Park MK, Park SA, Son SK, Park WW, Choi SH: Clinical and radiological outcomes of unilateral biportal endoscopic lumbar interbody fusion (ULIF) compared with conventional posterior lumbar interbody fusion (PLIF): 1-year follow-up. Neurosurg Rev 2019, 42 (3): 753-761.

[15] Almeida ND, Lee R, Bestourous D, Klein AL, Parekh NR, Sack K, Sherman JH: Perioperative Complications Associated with Severity of Anemia in Geriatric Patients Undergoing Spinal Procedures. World Neurosurgery 2020, 135.

[16] Li BH, Yu ZJ, Wang CY, Zi H, Li XD, Wang XH, Ren XY, Liu TZ, Zheng H: A Preliminary, Multicenter, Prospective and Real World Study on the Hemostasis, Coagulation, and Safety of Hemocoagulase Bothrops Atrox in Patients Undergoing Transurethral Bipolar Plasmakinetic Prostatectomy. Front Pharmacol 2019, 10: 1426.

[17] Roman-Dominguez L, Neri-Castro E, Vazquez Lopez H, Garcia-Osorio B, Archundia IG, Ortiz-Medina JA, Petricevich VL, Alagon A, Benard-Valle M: Biochemical and immunochemical characterization of venoms from snakes of the genus Agkistrodon. Toxicon X 2019, 4: 100013.

[18] Nogami K, Matsumoto T, Sasai K, Ogiwara K, Arai N, Shima M: A novel simultaneous clot-fibrinolysis waveform analysis for assessing fibrin formation and clot lysis in haemorrhagic disorders. Brit J Haematol 2019, 187 (4): 518-529.

[19] Jin FG, Li Q, Bai C, Wang HW, Li SY, Song Y, Zeng YM, Zhou R, Li WP, Hu CP et al: Chinese Expert Recommendation for Diagnosis and Treatment of Massive Hemoptysis. Respiration 2020, 99 (1): 83-92.

[20] Nagabhushan RM, Shetty AP, Dumpa SR, Subramanian B, Kanna RM, Shanmuganathan R: Effectiveness and Safety of Batroxobin, Tranexamic Acid and a Combination in Reduction of Blood Loss in Lumbar Spinal Fusion Surgery. Spine (Phila Pa 1976) 2018, 43 (5): E267-E273.

[21] de Barros E, Goncalves RM, Cardoso MH, Santos NC, Franco OL, Candido ES: Snake Venom Cathelicidins as Natural Antimicrobial Peptides. Frontiers in Pharmacology 2019, 10.

[22] Abd El-Aziz TM, Soares AG, Stockand JD: Snake Venoms in Drug Discovery: Valuable Therapeutic Tools for Life Saving. Toxins 2019, 11 (10). 